\title{
ARTICLES
}

\section{La nature du commentaire explicatif dans la traduction des toponymes et des anthroponymes - étude comparative}

\author{
The nature of explication commenatry in translation \\ of toponyms and anthroponyms - comparative study
}

\author{
Paulina Borowczyk \\ Université Adam Mickiewicz, Poznań \\ pborow@amu.edu.pl
}

\begin{abstract}
In the following article we will present the results of our study on the translation of toponyms and anthroponyms for instance at Arte TV news. First, we will introduce and define the notion of explication, one of translation procedures. And then, we will show the semantic content of explication commentary in translation of the names of people and places from French into German and vice versa. Our study has a comparative nature.
\end{abstract}

Keywords: translation of toponyms and anthroponyms, Franco-German channel Arte, translation procedures

\section{INTRODUCTION}

Dans le présent article, nous tenons à présenter les résultats partiels de l'étude comparative qui est centrée sur les différentes façons de traduction des noms propres, et plus particulièrement des toponymes et des anthroponymes. Travaillant sur le corpus défini et homogène constitué du même type d'émissions, c'est-à-dire le 
journal télévisé ${ }^{1}$ diffusé chaque soirée sur la chaîne de télévision franco-allemande Arte, notre objectif principal sera de décrire d'abord la technique de traduction qui est appliquée le plus souvent par les traducteurs audiovisuels, notamment l'ajout d'un commentaire explicatif à côté d'un nom propre original ${ }^{2}$. Ensuite, à l'aide des exemples convenables, nous visons à montrer quelle est la nature, c'est-à-dire le type de contenu sémantique dans les informations supplémentaires que les traducteurs ont jugées indispensables à la compréhension du texte donné par les récepteurs étrangers. On va observer donc les cas où les traducteurs préservent un terme original (français ou allemand) dans le texte d'arrivée et décident d'ajouter des expliactions pour son sens.

Nous divisons notre article en deux principales parties : la première où nous définissons la notion de commenatire explicatif et la deuxième qui comprend l'ensemble des exemples illustrant cette technique de traduction. L'analyse qui a un caractère comparatif se situe au niveau sémantique où nous étudions le contenu sémantique véhiculé par les informations ajoutées dans le texte d'arrivée et les conséquences qui en découlent pour le public cible. Les abréviations TD et TA renvoient respectivement au texte de départ et au texte d'arrivée, issus, tous les deux, des journaux télévisés.

À la fin, nous allons situer le commentaire explicatif que nous discutons dans cet article par rapport à d'autres techniques observées sur un corpus plus vaste. Cela nous permettra d'un côté de voir quelle est la fréquence de l'emploi du commentaire explicatif par les traducteurs dans le corpus analysé (109 exemples au total) et de l'autre, d'observer les tendances plus générales quant aux comportements des traducteurs lors du transfert des noms des réalités étrangères dans le contexte audiovisuel.

\footnotetext{
${ }^{1}$ Le journal télévisé d'Arte est un type d'émission dans laquelle on a affaire à deux méthodes de transfert audiovisuel, à savoir l'interprétation simultanée pour les contributions d'une / un journaliste et la voice-over pour les actualités développées tout au longu du JT.

${ }^{2}$ Les résultats partiels présentés dans le présent article font partie d'une grande étude que nous avons menée sur un un corpus constitué des émissions qui ont été enregistrées du 15 au 30 avril 2002 et comprenant en majeure partie les élections régionales en Allemagne, le premier tour de la présidentielle en France et les résultats approximatifs des scrutins. Le nombre total d'exemples provenant de notre corpus est de 109. Il s'agit des noms désignant des réalités étrangères, tantôt allemandes, tantôt françaises. 91 d'entre eux, ce qui constitue $83 \%$ de la totalité des cas relevés, appartiennet à la catégorie des noms propres. La conclusion générale tirée de cette analyse est telle que lors du transfert des noms des réalités culturelles appartenant à un pays concret les traducteurs laissent les termes étrangers dans leur forme originale et introduisent dans les textes cibles des renseignements additionnels qui dans le contexte donné sont nécessaires pour la compréhension d'un message. En plus, les techniques d'adaptation et de suppression des termes étrangers sont marginales dans le corpus étudié. À la fin de cet article, dans le bilan général, nous présentons les conclusions globales de l'étude (Paulina Borowczyk, Stratégies des traducteurs audiovisuels face aux réalités socioculturelles sur l'exemple de la télévision Arte, UAM, Poznań 2005).
} 


\section{LE COMMENTAIRE EXPLICATIF}

C'est un truisme de constater que chaque type de texte rédigé dans une langue donnée est bien marqué par la spécificité culturelle. Il s'agit des mots ou des expressions qui désignent des réalités bien connues par des récepteurs d'un texte de départ mais inconnues et le plus souvent démunies de sens pour des récepteurs étrangers. Comme le remarque Wojtasiewicz chaque allusion évoque les sentiments voulus par l'auteur uniquement chez des récepteurs qui la comprennent. Dans le cas des textes traduits la probabilité de l'incompréhension d'une allusion augmente considérablement parce que les récepteurs d'un texte d'arrivée ne disposent pas tout simplement de mêmes connaissances indispensables à la compréhension de l'original. De manière générale, le traducteur peut expliquer une allusion aux récepteurs étrangers mai il ne peut pas, selon nous, la traduire car sa formulaion ne sera pas adéquate à la formulation originale et n'évoquera pas chez des récepteurs d'un texte traduit les mêmes réactions que celles qui surgissent chez des lecteurs de départ (1992, p. 77-78).

La traduction des éléments culturels constitute donc un vrai défi pour les traducteurs. La majeure difficulté réside dans le fait que dans la langue d'arrivée il manque de termes pour désigner la réalité de l'Autre. Pour y rémedier, les traducteurs ont à leur disposition tout un éventail de procédés de traduction dont ils peuvent se servir lorsqu'ils sont confrontés aux termes culturellement connotés. On partage l'opinion de Skibińska qu'à partir de la moitié du XXe siècle « le rôle du traducteur est clairement défini comme celui du médiateur entre les langue et les cultures, celui du passeur de cultures, qui doit présenter l'Autre, l'Étranger, aux siens, membres de la société à laquelle il appartient, et assurer, par son activité, la communication interculturelle »(1999, p. 244). Même si cette constatation a été formulée en référence aux textes littéraires, on peut l'appliquer à d'autres contextes, d'autres situations où on affaire à la traduction. Face à la coopération permanante des chaînes de télévision de différents pays et à l'accès facile et direct aux actualités diffusées en continu sur les sites Internet, cette communication entre les cultures est possible grâce aux traducteurs qui rendent compréhensibles les textes originaux.

L'une des solutions employées par les traducteurs est la technique d'explication consistant « à introduire dans le texte d'arrivée, pour plus de clarté ou en raison de contraintes imposées par la langue d'arrivée, des précisions sémantiques non formulées dans le texte de départ, mais qui se dégagent du contexte cognitif ou de la situation décrite » (Delisle et al., 1999, p. 37). Pour illustrer ce procédé, Tomaszkiewicz fournit des exemples suivants :

- « Random House - aux éditions Random House w wydawnictwie Random House ; 
- You look like you stepped right of Life magazine

On te dirait sortir d'une magazine Life 1960

Jakbyś zeszła z okładki Life'u $1960 »$ (1996, p. 128) ;

- « w barze na Powiślu - dans un bar du quartier de Powiśle » (2004, p. 37).

Dans ces exemples, le traducteur a introduit dans les langues d'arrivée des informations supplémentaires pour que le récepteur du texte cible puisse comprendre l'élément culturel en question.

Dans la suite de l'article nous allons présenter les cas où les traducteurs, tout en laissant les éléments étrangers dans leur forme originale (les noms des lieux et des personnes) enrichissent le texte cible de quelques «précisions sémantiques » afin d'expliquer la réalité à laquelle renvoie un nom propre en question.

\section{LES TOPONYMES}

Du point de vue de l'importance des toponymes, on peut les subdiviser, selon Bagajewa (1992), en deux classes : les macro- et microtoponymes Macrotoponyms embrace three groups of names :

1) universal names, e.g. the names of the continents or oceans ;

2) regional names, such as the names of big seas, mountain ranges, large rivers and like, and

3) local names, which are a feature of one culture (unicultural) but which have such a broad cultural, political or historical scope that, in some form, they are present in at least several languages, as e.g. names of countries, capitals and large cities, large bodies of water, places of great historic importance etc. (p. 352).

Les microtoponymes renvoient à des noms de lieux de l'étendue locale, comme p.ex. les noms de petites villes, les noms de villages. Dans notre corpus, les deux classes de toponymes nous serviront d'exemples. Au niveau sémantique de l'analyse, nous avons relevé six façons d'introduire une explication à côté d'un toponyme. Chaque explication figurant à côté du nom propre en question véhicule un autre sens. Ainsi, le commentaire explicite le référent en indiquant :

- la classe d'objets à laquelle un toponyme appartient (ex. 1, 2, 3 et 4) ;

- le rapport entre un toponyme et un individu (ex. 5) ;

- la caractère d'un toponyme (ex. 6) ;

- la localisation spatiale d'un toponyme (ex. 7 et 8) ;

- aussi bien la classe d'objet que la localisation spatiale d'un toponyme (ex. 9). Indiquant le type de classe auquel appartient le toponyme étranger, les ajouts pratiqués par le traducteur dans quatre premiers exemples ont pour but d'expliciter le référent de celui-ci : 
1

\begin{tabular}{|l|l|}
\hline \multicolumn{1}{|c|}{ Texte français (TD) } & \multicolumn{1}{|c|}{ Texte allemand (TA) } \\
\hline $\begin{array}{l}\text { Images + commentaire off : } \\
\text { - «Quant à Lionel Jospin le Premier ministre, il } \\
\text { a accompli son devoir civique à Cintegabelle, en }\end{array}$ & $\begin{array}{l}\text { « Premierminister Lionel Jospin erfüllte seine } \\
\text { staatsbürgerlichen Pflichten im Departement } \\
\text { Haute-Garonne » (21.04.02). }\end{array}$ \\
\hline
\end{tabular}

Dans le premier exemple, le traducteur s'est servi de l'emprunt à la langue française, à savoir "das Departement». Ce dernier, figurant à côté du toponyme français explicite le référent en indiquant une catégorie qui n'est propre qu'à la culture de départ et que les auditeurs allemands n'ont pas ${ }^{3}$. Sans être entré dans le détail, le traducteur a supprimé dans le TA le nom du fief électoral de Lionel Jospin. Nous sommes d'avis que ce nom de localité est d'une importance secondaire par rapport au sens véhiculé par l'ensemble du message.

De même que dans le commentaire verbal, dans les notes en bas de l'écran, le traducteur explique au public allemand, à l'aide d'un emprunt à la langue française, à quelle réalité renvoie le toponyme étranger.

2

\author{
Dominique Barrau \\ Président de la FDSEA de l'Aveyron \\ Präsident Bauerngewerkschaft FDSEA, Departement Aveyron
}

Daniel Bouiller

Lieutenant gendarmerie du Tarn

Gendarmerie Departement Tarn

On s'est demandé ce que les Allemands apprenaient en lisant les sous-titres cidessus. D'une part, l'introduction de cet emprunt leur fait apprendre (à ceux qui ne le savent pas déjà) que Aveyron est le nom du département et de l'autre, elle leur permet d'éliminer d'autres possibilités d'interprétation, p.ex. qu'il s'agit du nom d'une ville. Ceci pourrait signifier qu'il s'agit moins de constituer un savoir que de proposer une classification grossière qui va permettre au spectateur allemand une toute première catégorisation dont les journalistes et les traducteurs pensent probablement qu'il a besoin pour comprendre le texte. C'est entre autres à travers le commentaire ajouté à côté du nom propre que le traducteur rend réceptible le texte d'arivée.

\footnotetext{
${ }^{3}$ Dans notre article intitulé «Transferts culturels à la télévision franco-allemande Arte », à part la technique d'explication, nous présentons tous les procédés employés lors de la traduction des réalités étrangères dans les journaux télévisés bilingues (Studia Romanica Posnaniensia, vol. XXXIV, Wydawnictwo Naukowe UAM, Poznań 2007).
} 
De plus, l'emploi du nom propre original préserve la couleur et l'identité socioculturelle de l'autre pays. «La préservation du signifiant est comme une concession à l'étranger, et contribue, du point de vue de la réception, à la création de la couleur locale » (Ballard, 2001, p. 27). Folkart (1986) désigne ce phénomène comme une « rupture d'isoglossie » (p. 234). " Même le nom propre choisi au départ pour sa banalité s'auréole dans le texte-cible du connoté étranger [...] du seul fait de se trouver en rupture avec un co-texte qui lui est désormais hétéroglosse » (p. 234). C'est donc grâce à un traducteur - un intermédiaire entre deux cultures que le lecteur d'un TA reçoit une image de la culture d'origine. « Ce que le lecteur ignorait de la culture étrangère, il l'apprend en lisant» (Lederer, 1994, p. 127) ou bien en l'écoutant pendant la diffusion des émissions télévisuelles.

Si on regarde l'exemple qui suit, on aurait pu croire qu'il joint la logique des cas qui l'ont précédé et qu'il n'en diffère pas. Pourtant, une erreur de fond concernant la division administrative française a été commise dans le texte cible.

3

\begin{tabular}{|l|l|}
\hline \multicolumn{1}{|c|}{ Texte français (TD) } & \multicolumn{1}{c|}{ Texte allemand (TA) } \\
\hline Le présentateur : & \\
- « Après les réactions internationales, tentons & • Nach den internationalen Reaktionen nun zu \\
à présent un début d'explication du scrutin. & den Erklärungsversuchen. Wie konnte es dazu \\
Comment a-t-on pu en arriver là? Un seul & kommen? Nur ein Beispiel : das Departement \\
exemple suffit, celui du Nord-Pas-de-Calais, un & Nord-Pas-de-Calais im Norden Frankreichs war \\
bastion traditionnel de la gauche. Jusqu'à hier en & ja traditionell eine Hochburg der Linken. Und \\
tout cas, car dans les deux départements, les & das hat sich spätestens seit gestern geändert. \\
électeurs ont placé Jean-Marie Le Pen en tête. & $\begin{array}{l}\text { Merklich gaben die Menschen dort ihre Stimme } \\
\text { dem rechtspopulistischen Kandidaten Jean-Marie }\end{array}$ \\
L'exemple de Calais illustre ce basculement de & Le Pen. So in der Stadt Calais ». \\
l'électorat » (22.04.02). & \\
\hline
\end{tabular}

L'objectif du traducteur était sans doute de faire apprendre au public allemand à quoi se réfère le nom propre «Nord-Pas-de-Calais». Or, le terme cité renvoie à une région administrative du Nord de la France, constituée de deux départements : le Nord et le Pas-de-Calais, ce que l'on peut d'ailleurs déduire de la suite du texte de départ. Même si les deux noms de départements considérés séparément n’y sont pas évoqués, on apprend qu'il y en a deux (" dans les deux départements »). Ce passage a été effacé dans le texte d'arrivée et remplacé par l'adverbe « dort» («là-bas »). Par conséquent, un spectateur allemand peut faussement en conclure que le NordPas-de-Calais est effectivement un département français et non une région comptant deux départements.

Dans les exemples suivants, c'est par le biais d'une description de type encyclopédique raccourcie que le sens des noms propres est véhiculé dans le TA : 
$3 / 4$

\begin{tabular}{|c|c|}
\hline Texte français (TD) & Texte allemand (TA) \\
\hline $\begin{array}{l}\text { Le présentateur: } \\
\text { - « Nos reporters de la Méridienne sont ce soir } \\
\text { dans le berceau du socialisme. A Carmaux, dans } \\
\text { le Tarn, un département profondément ancré } \\
\text { à gauche. Carmaux, c'est ici que Jean Jaurès } \\
\text { s'est lancé dans la politique »(17.04.02). } \\
\text { - [...] L'exemple de Calais illustre ce basculement } \\
\text { de l'électorat» (22.04.02). }\end{array}$ & $\begin{array}{l}\text { - «Unser Team vom grünen Meridian berichtet } \\
\text { heute aus dem Departament Tarn, am gleichna- } \\
\text { migen Fluß. Hier wählt man traditionell links. In } \\
\text { der Stadt Carmaux ist nämlich Jean Jaurès in die } \\
\text { Politik gegangen ». } \\
\text { - « [...] So in der Stadt Calais ». }\end{array}$ \\
\hline
\end{tabular}

En attribuant respectivement aux toponymes français « Carmaux » et "Calais » le substantif « die Stadt» (la ville), on procède par une classification des référents auxquels ils renvoient.

Dans l'exemple suivant, c'est la relation entre le nom de candidat à la présidentielle et le nom de lieu qui est soulignée dans le développement définitionnel figurant à côté du toponyme même :

5

\begin{tabular}{|c|c|}
\hline Texte français (TD) & Texte allemand (TA) \\
\hline $\begin{array}{l}\text { Images + commentaire off: } \\
\text { - « [...] Le président sortant Jacques Chirac a voté } \\
\text { à Saran, son fief de Corrèze, en compagnie de } \\
\text { son épouse Bernadette }(21.04 .02) \text {. }\end{array}$ & $\begin{array}{l}\text { - « [...] Noch Amtsinhaber Chirac wählte in sei- } \\
\text { nem Wahlkreis Saran, in Begleitung von Gattin } \\
\text { Bernadette. }\end{array}$ \\
\hline
\end{tabular}

Ici, l'explication du référent apparaît sous forme d'un élément de contextualisation informant le spectateur allemand que Saran est la circonscription électorale de Jacques Chirac ( « in seinem Wahlkreis »). Vu que c'est le moment de l'élection présidentielle en France et le jour de vote même, le traducteur a préféré mettre en relief le rapport entre Jacques Chirac présent sur l'écran et Saran. Dans ce cas, le développement définitionnel ne fait pas ranger le toponyme dans une classe d'objet mais il en fait apparaitre d'autres informations : Saran est un lieu (le public allemand ignore s'il s'agit d'une ville, d'une région, d'une commune ou d'un département) où Chirac est allé voter.

On partage l'avis de Levy (1979) cité par Brown et Yule (1983) que «le locuteur, en référant, n'identifie pas seulement, mais construit un objet, en sélectionnant d'un champ relationnel les propriétés qui sont pertinentes au moment de l'énonciation » (Levy, 1979; Brown et Yule, 1983, as cited in Jonasson, 1994). Il 
s'en suit pour la traduction que les explications fournies par le traducteur à côté des noms propres peuvent varier d'un contexte à l'autre. Ce qui est saillant dans un contexte, ne le serait pas dans l'autre. Ainsi, dans notre contexte, il est pertinent de parler de «Wahlkreis » tandis que dans un autre, le traducteur aurait évoqué d'autres traits caractéristques de Saran. Cette description du référent a été jugée utile par le traducteur afin d'assurer au public allemand non seulement la réussite de la référence au particulier visé, mais surtout la bonne interprétation et la cohérence du discours télévisuel.

Dans l'exemple ci-dessous le traducteur a préféré souligner la destination du bâtiment parisien et le caractère des événements qui y ont lieu (« das Kulturzentrum / le centre de culture $»)$.

6

\begin{tabular}{|c|c|}
\hline Texte français (TD) & Texte allemand (TA) \\
\hline $\begin{array}{l}\text { Le présentateur: } \\
\text { - « Et cette réaction du monde de la culture, c'est } \\
\text { aussi l'un des aspects de la mobilisation en } \\
\text { France. Plusieurs centaines d'artistes se sont } \\
\text { rassemblés devant le Centre Pompidou à Paris et } \\
\text { ils ont clairement appelé à voter Jacques Chirac } \\
\text { dimanche prochain ». }\end{array}$ & $\begin{array}{l}\text { - «Auch in Frankreich engagieren sich Künstler } \\
\text { gegen Rechts. Mehrere hundert von ihnen ver- } \\
\text { sammelten sich heute vor dem Pariser Kultur- } \\
\text { zentrum Georges Pompidou, um für Sonntag zur } \\
\text { Wahl von Jacques Chirac aufzurufen ». }\end{array}$ \\
\hline
\end{tabular}

Dans son analyse des toponymes, Ballard ramène l'étymologie de ceux-ci à deux grands schémas, à savoir « la dérivation à partir d'un éponyme et l'utilisation d'une appellation ou d'un syntagme descriptifs » (2001, p. 121). Dans le cas présent, le toponyme «le Centre Pompidou» (dont le nom entier est le «Centre national d'art et de culture Georges Pompidou ; C.N.A.C ») fonctionne à partir d'un anthroponyme préexistant renvoyant à l'homme politique qui était un grand admirateur de l'art moderne et le promoteur du lieu, en l'honneur de qui les Français ont nommé le centre, et le nom commun masculin «le centre ». On peut observer dans le cas présent que le texte d'arrivée a été enrichi d'un substantif «Kultur». Ce dernier, faisant partie du mot composé «Kulturzentrum», met en relief le caractère de l'établissement en question. De plus, on peut y observer le changement de catégorie et de fonction. Le groupe prépositionnel français « à Paris » employé en fonction de complément circonstanciel de lieu est rendu en allemand par un adjectif indéclinable « Pariser » qui est un dérivé du nom de la capitale française « Paris ». Il s'emploie en allemand en fonction d'épithète.

Dans les exemples qui suivent, c'est la localisation spatiale des toponymes qui est mise en relief dans le TA : 
7

\begin{tabular}{|l|l|}
\hline \multicolumn{1}{|c|}{ Texte français (TD) } & \multicolumn{1}{c|}{ Texte allemand (TA) } \\
\hline Images + commentaire off : & \\
$\bullet$ « Le bureau des élèves de l'École des Ponts et & $\bullet$ «Der Aufenthaltsraum der Studenten an der Pa- \\
$\begin{array}{l}\text { Chaussées, l'une des plus anciennes et des plus } \\
\text { prestigieuses écoles d'ingénieurs de France. Ste- }\end{array}$ & $\begin{array}{c}\text { riser École des Ponts et Chaussées, einer der } \\
\text { ältesten und angesehensten Ingenieurschulen }\end{array}$ \\
$\begin{array}{l}\text { phanie Luh y est assistante d'allemand. Son manque pas d'élèves. Que ce soit en } \\
\text { première, deuxième ou troisième langue, le nom- }\end{array}$ & $\begin{array}{l}\text { Frankreichs. Stephanie Luh ist Deutschreferen- } \\
\text { darin. In ihrem Kurs sind genügend Schüler, ob }\end{array}$ \\
$\begin{array}{l}\text { Deutsch erste, zweite oder dritte Fremdsprache } \\
\text { bre de germanistes non seulement ne baisse pas } \\
\text { mais il augmente » (18.04.02). }\end{array}$ & $\begin{array}{l}\text { ist. Die Anzahl der Germanisten sinkt nicht, sie } \\
\text { steigt ». }\end{array}$ \\
\hline
\end{tabular}

Ici, le traducteur s'est servi dans le texte cible d'un adjectif «Pariser» afin d'expliquer au public allemand où se trouve l'école d'ingénieurs dont il est question dans la suite du reportage. C'est de ce dérivé du nom de la capitale française « Paris » que le groupe prépositionnel allemand a été élargi.

S'inscrivant dans la logique des exemples précédents, dans ce qui suit, le traducteur a précisé dans le TA dans quel pays se trouve Ramstein, la base des soldats américaine.

8

\begin{tabular}{|l|l|}
\hline \multicolumn{1}{|c|}{ Texte allemand (TD) } & \multicolumn{1}{c|}{ Texte français (TA) } \\
\hline Images + commentaire off : & \\
- «Zwei schwer verletzte und vier leicht verletzte & $\bullet$ « Arrivés sur la base américaine de Ramstein, en \\
kanadische Soldaten sind am späten gestrigen & $\begin{array}{l}\text { Allemagne, des soldats canadiens blessés mer- } \\
\text { Abend am amerikanischen Luftwaffenstützpunkt } \\
\text { credi par un bombardement américain en Afgha- } \\
\text { Ramstein eingetroffen » (19.04.02). }\end{array}$ \\
\hline
\end{tabular}

Dans le cas suivant, le développement définitionnel indique aussi bien la classe d'objet que la localisation spatiale du toponyme allemand.

9

\begin{tabular}{|l|l|}
\hline Texte allemand (TD) & Texte français (TA) \\
\hline $\begin{array}{l}\text { Le présentateur : } \\
\text { • « Am Sonntag wird in Sachsen-Anhalt gewählt } \\
\text { und die Republik wird das mit Spannung verfol- } \\
\text { gen » (19.04.02). }\end{array}$ & $\begin{array}{l}\text { « Tout le pays aura les yeux rivés sur la Saxe- } \\
\text { Anhalt, un land de l'Est plutôt mal au point où } \\
\text { différentes alliances pourraient suivre le scrutin ». }\end{array}$ \\
\hline
\end{tabular}

Ici, le traducteur a utilisé l'emprunt à la langue allemande «land» afin d'expliquer aux spectateurs français le nom propre "Sachsen-Anhalt», référant à l'un des 16 états, appelés «Länder» qui forment le pays. De plus, il a décidé de 
préciser dans quelle partie de l'Allemagne se situe le land en question (« un land de l'Est »).

La traduction des toponymes fait apparaitre leur forte charge sémantique. Celleci « réside dans une réalité extralinguistique perçue comme caractéristique d'une culture et distincte de celle de la culture réceptrice, celle pour laquelle on traduit. $\mathrm{Ce}$ qui bloque la traduction n'est pas l'absence de sens mais la relation forte qui unit un signifiant spécifique à une réalité unique et spécifique » (Ballard, 2001, p. 120). Les exemples cités montrent que le sens est véhiculé par les traducteurs à travers l'introduction d'un commentaire explicatif à côté des toponymes dans le TA. Nous avons vu qu'au niveau sémantique les éléments explicatifs peuvent transmettre des informations différentes.

\section{LES ANTHROPONYMES}

Relativement aux anthroponymes, K. Jonasson distingue trois types de noms propres, à savoir : historiques, familiers et de l'actualité. Le référent du premier type des Npr « est donc une personne célèbre, dont la connaissance fait partie du patrimoine culturel de la communauté linguistique donnée. Ces noms propres sont 'culturellement saillants' pour emprunter une expression que Wierzbicka (1985) applique aux noms communs. Cette saillance culturelle des noms propres historiques a pour corollaire l'existence, dans la mémoire collective de la communauté linguistique, d'un référent 'par défaut', référent qu'on trouve associé au nom propre dans les encyclopédies et les dictionnaires des noms propres » (Jonasson, 1994, p. 138). Pour illustrer ce type, l'auteur cite des exemples tels que Socrate, Jeanne d'Arc ou Napoléon. Leur interprétation se distingue de celle des noms propres familiers, pour lesquels "aucun référent 'par défaut' n'existe au niveau de la mémoire collective de la communauté linguistique. Ceux-ci sont souvent associés à de nombreux particuliers dans la communauté linguistique, mais désignent dans un champ restreint (famille, classe, bureau, études, vie privée, village, etc.) un seul ou un nombre limité de particuliers » $(1994$, p. 138). Ils renvoient donc à un référent saillant à l'intérieur d'une sphère privée. On voit bien que ces deux premiers types de noms propres se retrouvent plutôt «aux extrémités d'une échelle allant de la célébrité ou la notoriété à la familiarité ou l'intimité » (1994, p. 138).

On partage l'avis de l'auteure qu'entre ces deux extrêmes, il existe toute sorte de noms propres intermédiaires qui exigent pour leur interprétation des connaissances sur des événements d'actualité, des relations humaines, des fonctions sociales et professionnelles, etc. C'est pour cette raison que Jonasson a introduit un troisième type de noms propres, à savoir les noms propres d'actualité. Il s'agit de noms propres qui « ne sont pas encore devenus historiques mais dont les porteurs occupent un rôle social dans l'actualité contemporaine et qui sont donc culturellement 
saillants. Nous voulons parler de nomsa propres qui remplissent les pages de jour naux, qu'ils soient quotidiens, hebdomadaires ou mensuels, et qui désignent des personnages qui se font remarquer d'une manière ou d'une autre [...] » (p. 148). Dans les journaux télévisés analysés, presque la totalité des anthroponymes peuvent être classés «Npr d'actualité ». Ceux-ci renvoient surtout à des personnages exerçant une fonction politique, culturelle, professionnelle ou médiatique dans le monde actuel. Pourtant, nous pensons que dans certains cas, il est difficile de classifier définitivement des porteurs de nom propre à une seule catégorie (soit à la catégorie de noms propres historiques soit à celle de noms propres d'actualité). Si l'un des critères est que l'on peut trouver le référent associé au Npr dans des encyclopédies, des personnages tels que Gerhard Schröder, Lionel Jospin ou Jacques Chirac appartiendraient à la catégorie de noms propres historiques, ce qui serait juste du point de vue de rôle qu'ils jouent sur la scène politique nationale et / ou mondiale.

Dans les exemples qui suivent, en gardant le prénom et le nom d'origine, le texte cible est en plus enrichi de descriptions supplémentaires, absentes dans le texte original. On est d'avis que les renseignements donnés, que ce soit dans le texte source ou dans le texte cible, sont ceux que le journaliste et ensuite le traducteur ont jugé indispensables à la construction d'un objet de référence et à la bonne interprétation du discours dans lequel figure le nom propre. Or, le rôle qu'ils jouent est différent dans le TD et le TA.

Le commentaire explicite donc le référent en indiquant :

- aussi bien le titre que la nationalité d'une personne (ex. 1,2 et 4);

- la fonction exercée par une personne (ex. 3);

- la relation entre deux individus (ex. 5);

- la fonction qu'une personne a l'intention d'exercer (ex. 6) ;

- aussi bien la fonction qu'une personne exerce sur la scène politique que le caractère de parti qu'elle représente (ex. 7);

- le caractère du mouvement politique qu'une personne représente (ex. 8 et 9).

Dans les deux premiers cas, le traducteur a mis en relief dans le TA aussi bien la nationalité que le titre des référents :

$1 / 2$

\begin{tabular}{|l|l|}
\hline \multicolumn{1}{|c|}{ Texte français (TD) } & \multicolumn{1}{|c|}{ Texte allemand (TA) } \\
\hline Le présentateur: & \\
$\bullet$ « Lionel Jospin a tiré aujourd'hui un trait final & $\bullet$ «Der französische Staatschef Chirac und Pre- \\
sur cinq ans de cohabitation forcée avec Jacques & mierminister Lionel Jospin haben heute einen \\
Chirac. Au cours du dernier Conseil des mini- & Schlussstrich unter ihre erzwungene politische \\
stres de ce mandat, ce matin à l'Elysée, Lionel & Zusammenarbeit gezogen. Am Vormittag fand \\
Jospin a remercié l'ensemble des ministres pour & der letzte Ministerrat der insgesamt 5-jährigen \\
le travail accompli. Il a également salué la cour- & Kohabitation statt. Jospin dankte seinen Kabi- \\
toisie avec laquelle Jacques Chirac avait conduit & nettsmitglieder und Chirac für die geleistete \\
pendant cinq ans les travaux du Conseil des & Arbeit». \\
ministres» $»(24.04 .02)$. & \\
\hline
\end{tabular}


Dans ces deux cas, le traducteur a expliqué, tout au début de l'information adressée au public allemand, qui sont les deux hommes dont les noms sont cités dans la suite de l'actualité. De plus, le traducteur a précisé aussi bien leur nationalité (« der französische») que leur titre («Staatschef / Premierminister »). Le titre marque ainsi l'autorité et le prestige de la personne. Il est à remarquer qu'une fois le référent des noms propres expliqué dans le TA, ceux-ci sont utlisés tels quels dans la suite de l'actualité (Chirac / Jospin).

Dans l'exemple suivant, les éléments ajoutés dans le TA indiquent la fonction exercée par les hommes politiques allemands :

3

\begin{tabular}{|c|c|}
\hline Texte allemand (TD) & Texte français (TA) \\
\hline $\begin{array}{l}\text { Images + commentaire off: } \\
\text { - «[...] 17\% Verlust musste SPD-Kandidat Rein- } \\
\text { hard Höppner einstecken. Eine herbe Enttäu- } \\
\text { schung hat Sachsen-Anhalt ihm damit beschei- } \\
\text { nigt. Er hat sofort seine Rückzug aus der Lan- } \\
\text { despolitik angekündigt. Besonders gelitten aber } \\
\text { hat die SPD unter der dramatisch schlechten } \\
\text { Wahlbeteiligung, nur etwa 55\% der Anhaltiener } \\
\text { gingen wählen. Nach Meinungsumfragen soll } \\
\text { ein Dritter der } 2,1 \text { Millionen Wahlberechtigten } \\
\text { noch vor einer Woche gar nicht gewußt haben, } \\
\text { dass überhaupt Wahlen stattfänden. Immerhin } \\
15 \% \text { mehr aber konnten sich dazu durchdringen, } \\
\text { den Kandidaten der CDU zu wählen. Wolfgang } \\
\text { Böhmer hat einen Sieg hingelegt, den man in } \\
\text { Berlin gerne nachmachen würde » (21.04.02). }\end{array}$ & $\begin{array}{l}\text { - « [...] Les grands perdants, les voilà : les sociaux- } \\
\text { démocrates. C'est une vraie déroute que doit } \\
\text { encaisser Reinhard Höppner, leur chef de file dans } \\
\text { ce land. Le parti du chancelier a perdu plus de la } \\
\text { moitié de son électorat en quatre ans. Et puis, le } \\
\text { mal n'est pas exclusivement français. Outre-Rhin } \\
\text { aussi, l'abstention a fait un carton. 55\% seulement } \\
\text { de deux millions d'électeurs se sont déplacés. } \\
\text { D'après les sondages, la semaine dernière encore } \\
\text { un tiers d'entre eux déclarait ne même pas savoir } \\
\text { qu'il y avait des élections. Mais ceux qui ce sont } \\
\text { déplacés ont offert un triomphe à l'opposition } \\
\text { chrétienne-démocrate. La CDU locale et son chef } \\
\text { l'emportent haut la main et à Berlin, on rêve de } \\
\text { renouveler l'exploit en septembre». }\end{array}$ \\
\hline
\end{tabular}

Les descritpions du référent (Reinhard Höppner) sont présentes aussi bien dans le TD que le TA. Dans le texte allemand, les journalistes évoquent que Reinhard Höppner représente le parti SPD (SPD-Kandidat) tandis que dans le texte français le traducteur explique aux spectateurs d'arrivée quelle place tient l'homme politique allemand au sein de son parti social-démocrate SPD, au pouvoir jusqu'au avril 2002 en Saxe-Anhalt. Ils apprennent alors que Höppner est le leader de ce parti. Et comme le traducteur a procédé au début du TA par la description du sigle allemand SPD (les sociaux-démocrates), l'adjectif possessif leur indique l'appartenance de Höppner à ce mouvement. Il s'établit ainsi une relation entre ce qui est « possédé » et celui dont on parle : leur chef de file. Ensuite, supprimant le nom de Wolfgang Böhmer dans le texte français, le traducteur n'a évoqué que la fonction exercée par celui-ci au sein de la CDU.

Il arrive aussi que dans le même extrait on ait affaire à deux types d'explication des référents : 
$4 / 5$

\begin{tabular}{|c|c|}
\hline Texte allemand (TD) & Texte français (TA) \\
\hline $\begin{array}{l}\text { Le présentateur: } \\
\text { - « Es ist die letzte Landtagswahl vor dem Sep- } \\
\text { tember. Von großer Koalition über ein Regie- } \\
\text { rungsbündnis mit der Schillpartei bis rot / rot ist } \\
\text { noch so ziemlich alles drin. Und es handelt sich } \\
\text { um eins der Sorgenkinder unter den deutschen } \\
\text { Bundesländern. Am Sonntag wird in Sachsen- } \\
\text { Anhalt gewählt und die Republik wird das mit } \\
\text { Spannung verfolgen. Der Landtagswahlkampf } \\
\text { wurde zum ersten Schaulaufen zwischen Schrö- } \\
\text { der und Stoiber. Sie übertrafen sich in den letz- } \\
\text { ten Wochen im Anpreisen von allen Heilrezep- } \\
\text { ten gegen Arbeitslosigkeit und Abwanderung im } \\
\text { Osten. Doch welche Partei wird überzeugen? » } \\
\text { (19.04.02). }\end{array}$ & $\begin{array}{l}\text { - «Ce dimanche auront lieu en Allemagne les der- } \\
\text { nières élections régionales avant les législatives } \\
\text { et la désignation du chancelier au mois de sep- } \\
\text { tembre. Tout le pays aura les yeux rivés sur la } \\
\text { Saxe-Anhalt, un land de l'Est plutôt mal en point } \\
\text { où différentes alliances pourraient suivre le scru- } \\
\text { tin. En Saxe-Anhalt, la campagne aura été l'occa- } \\
\text { sion d'un premier tour de piste pour le chancelier } \\
\text { Gerhard Schröder et son rival bavarois Edmund } \\
\text { Stoiber. Depuis des semaines, c'est à qui pro- } \\
\text { posera les meilleures recettes miracles contre le } \\
\text { chômage et l'exode dont souffrent les länder de } \\
\text { l'Est». }\end{array}$ \\
\hline
\end{tabular}

Le développement figurant à côté du premier Npr indique le titre du référent : le chancelier Gerhard Schröder. Comme il y est question entre autres des législatives, de la désignation du chancelier allemand et de la campagne électorale en cours, Edmund Stoiber apparaît en tant que rival bavarois de Gerhard Schröder, chancelier et en même temps candidat à la chancellerie. C'est la relation s'instaurant entre les deux hommes politiques qui est soulignée dans le TA.

Il arrive aussi que le même homme politique exerce plusieurs fonctions sur la scène politique, comme c'est le cas de Lionel Jospin : le Premier ministre français est également candidat aux élections présidentielles 2002 en France :

6

\begin{tabular}{|l|l|}
\hline \multicolumn{1}{|c|}{ Texte français (TD) } & \multicolumn{1}{c|}{ Texte allemand (TA) } \\
\hline Images + commentaire off : & \\
- «Il y a deux semaines le candidat Lionel Jospin & $\bullet$ « Vor zwei Wochen hat noch der Präsidentschaf- \\
avait fait un discours dans cette église. Il avait & tskandidat Lionel Jospin eine Rede im franzö- \\
réservé à Berlin sa dernière visite à l’étranger » & sischen Dom gehalten. Berlin war seine letzte \\
$(22.04 .02)$. & Wahlkampfstation im Ausland». \\
\hline
\end{tabular}

C'est le contexte qui détermine laquelle des deux références est la plus appropriée. Ici, Lionel Jospin s'est rendu à Berlin durant sa campagne électrorale plutôt en tant que candidat aux élections présidentielles que comme représentant du gouvernement français. Ainsi, c'est la fonction que Jospin a l'intention d'exercer qui a été mise en relief et jugée indispensable à la bonne interprétation du discours dans lequel figure le nom propre. 
Dans le cas suivant, le commentaire explicite le référent en indiquant aussi bien le rôle politique d'une personne que le caractère du parti dont elle est membre :

7

\begin{tabular}{|c|c|}
\hline Texte français (TD) & Texte allemand (TA) \\
\hline $\begin{array}{l}\text { Le présentateur: } \\
\text { - «Un seul exemple suffit, celui du Nord-Pas-de- } \\
\text { Calais, un bastion traditionnel de la gauche. } \\
\text { Jusqu'à hier en tout cas, car dans les deux } \\
\text { départements, les électeurs ont placé Jean-Marie } \\
\text { Le Pen en tête» (22.04.02). }\end{array}$ & $\begin{array}{l}\text { Nur ein Beispiel : das Departement Nord-Pas-de- } \\
\text { Calais im Norden Frankreichs war ja traditionell } \\
\text { eine Hochburg der Linken. Und das hat sich } \\
\text { spätestens seit gestern geändert. Merklich gaben } \\
\text { die Menschen dort ihre Stimme dem rechtspo- } \\
\text { pulistischen Kandidaten Jean-Marie Le Pen ». }\end{array}$ \\
\hline
\end{tabular}

Ici, on peut observer que le texte allemand a été enrichi par deux éléments lexicaux, absents dans le TD, l'adjectif rechtpopulistisch et le substantif der Kandidat. C'est à l'aide de l'adjectif rechtspopulistich que le traducteur décrit la ligne politique de Jean-Marie Le Pen, candidat à la présidence française et chef du parti d'extrême droite : le Front National. Le substantif der Kandidat souligne le fait que Le Pen prétend à la fonction du chef de l'Etat.

Les cas qui suivent montrent que le caractère d'un mouvement politique peut servir à décrire également les personnes y adhérant :

8

\begin{tabular}{|c|c|}
\hline Texte allemand (TD) & Texte français (TA) \\
\hline $\begin{array}{l}\text { Images }+ \text { commentaire off: } \\
\text { - « Mit dem Ziel, den Eltern die Wahl zwischen } \\
\text { Familie und Beruf zu lassen, will Kanzlerkan- } \\
\text { didat Stoiber für kleine Kinder } 600 \text { Euro Fami- } \\
\text { liengeld monatlich einführen }[\ldots] »(18.04 .02) \text {. }\end{array}$ & $\begin{array}{l}\text { - «L'opposition conservatrice veut donc laisser le } \\
\text { choix aux parents, et Edmund Stoiber, candidat } \\
\text { chrétien-démocrate à la chancellerie promet une } \\
\text { allocation familiale de } 600 \text { euros par mois pour } \\
\text { les enfants de moins de } 4 \text { ans ». }\end{array}$ \\
\hline
\end{tabular}

C'est de l'adjectif chrétien-démocrate décrivant l'orientation du parti allemand CDU dont Stoiber est le chef en Bavière que le TA a été élargi.

C'est aussi la ligne du parti politique qui est utilisée dans le cas suivant pour caractériser l'homme politique français : 
9

\begin{tabular}{|c|c|}
\hline Texte français (TD) & Texte allemand (TA) \\
\hline $\begin{array}{l}\text { Diffusion en direct du studio de Paris : } \\
\text { - « Ensuite, nous avons Bruno Mégret 2,4\%, ce } \\
\text { qui fait, si on aditionne le score de Jean-Marie } \\
\text { Le Pen à celui de Bruno Mégret, on a l'extrême } \\
\text { droite qui, si mes calculs sont bons, est quasi- } \\
\text { ment à égalité ou dépasse même Jacques Chirac » } \\
(21.04 .02) \text {. }\end{array}$ & $\begin{array}{l}\text { - « Bruno Mégret, der zweite Rechtsradikale mit } \\
\text { 2,4\%. Wenn man das zu dem Ergebniss von } \\
\text { Jean-Marie Le Pen noch hinzuzählt, ist die extre- } \\
\text { me Rechte bei diesen Präsidentschaftswahlen } \\
\text { ungefähr so stark wie der Präsident Jacques } \\
\text { Chirac». }\end{array}$ \\
\hline
\end{tabular}

$\mathrm{Vu}$ le caractère du mouvement auquel appartient Bruno Mégret: le MNR (Mouvement national républicain), c'est en ajoutant dans le texte cible un nom der Rechtsradikale (d'extrême droite) que le candidat à la présidence est présenté. De plus, l'adjectif «zweite» et le commentaire portant sur le score aditionné des deux candidats signalent que le parti de Bruno Mégret est la deuxième force de l'extrême droite en France. Or, le lien entre le parti de Le Pen et Mégret demeure inconnu pour le public allemand. Ce dernier ignore qu'à l'issue d'une discorde avec Jean-Marie Le Pen, Bruno Mégret est exclu du parti fin 1998 et fonde en 1999 le Mouvement national républicain qu'il préside depuis. Ceci pourrait expliquer pourquoi l'extrême droite est divisée en deux partis.

\section{BILAN PARTIEL}

On constate que le commentaire explicatif joint aux noms des lieux et des personnes permet à la traduction d'assumer pleinement sa fonction : la préservation du terme original dans le TA (introduction de la couleur locale) et l'identification du référent par le public cible (transfert du sens). Nous avons vu qu'au niveau sémantique, les éléments explicatifs peuvent véhiculer les différents contenus. Le fait d'introduire des explications supplémentaires à côté des toponymes et les anthroponymes connus dans la socioculture de départ révèle à quel point la perception et la compréhension d'un nom propre est importante pour les traducteurs. En plus, il faut souligner que la contrainte temporelle limite l'emploi des procédés à visée explicative et que les traducteurs doivent sélectionner des informations plus importantes de celles qui en sont moins dans le contexte donné. Pour ce qui est des messages audiovisuels" "le transfert linguistique ajoute des informations à celles contenues dans le texte original et en néglige d'autres. Il ne peut jamais tenter de restituer chaque atome d'information d'une langue dans une autre. Il s'agit plus ou moins d'une traduction classique. Moins car il ne traduit pas tout et plus parce que le traducteur-auteur audiovisuel doit prendre des décisions rédactionnelles permanentes 
en ce qui concerne les omissions ou la condensation du texte original, tandis que de nouvelles informations doivent être insérées » (Luyken et al., 1991, p. 167). Ne pas laisser le récepteur étranger en ignorance est la tâche principale du traducteur.

\section{BILAN GÉNÉRAL}

Après l'analyse de 109 termes renvoyant aux réalités socioculturelles provenant d'un contexte déterminé et de leurs traductions, nous pouvons en dégager trois stratégies principales : commençant par la préservation du terme étranger, sans recourir à une explication quelconque de celui-ci dans le texte d'arrivée $(42,20 \%)$, allant par la préservation du terme étranger et par l'ajout des explications pour son sens dans le texte d'arrivée $(47,70 \%$ - le cas analysé dans le présent article), et finissant par le gommage des références culturelles dans le texte d'arrivée : soit par l'emploi d'un équivalent culturel, soit par l'omission complète du terme d'origine $(10,09 \%)$. Les pourcentages cités démontrent que les proportions dans l'application de chacune de ces stratégies sont différentes. Il en résulte que dans la plupart des cas (dans 98 cas sur 109), le traducteur préserve les termes étrangers dans le texte d'arrivée et plus que la moitié de ceux-ci est explicitée. Relativement rares sont des cas où on gomme les termes relevant des réalités socioculturelles d'origine.

Étant donné que le journal télévisé est une émission d'informations dans laquelle on présente la synthèse de l'actualité, il n'est pas étonnant que dans la majorité des cas les traducteurs reportent tels quels les éléments socioculturels étrangers dans le texte d'arrivée et précisent à quoi ou à qui ils réfèrent. On ne remplace ni on ne supprime les termes relevant de la socioculture dans les textes à caractère informatif qui servent à forunir les traits caractèristiques de l'action réelle et contribuent considérablement à la construction de l'événement médiatique. 


\section{BIBLIOGRAPHIE}

Bagajewa, I. (1992). « Geographical names. Problems of equivalence and translatability ». Translation and Meaning, part 2, 349-354.

Ballard, M. (2001). Le nom propre en traduction. Paris : Ophrys.

Borowczyk, P. (2007). « Transferts culturels à la télévision franco-allemande Arte ». Studia Romanica Posnaniensia, XXXIV, 17-32.

Delisle, J., Lee-Jahnke, H., Cormier, M. (1999). Terminologie de la traduction. Amsterdam / Philadelphia : John Benjamins Publishing Company.

Folkart, B. (1986). Traduction et remotivation onomatsique. Meta, 31 (3), 233-252.

Jonasson, K. (1994). Le nom propre. Constructions et interprétations. Louvain-la Neuve : Editions Duclot.

Lederer, M. (1994). La traduction aujourd'hui. Modèle interprétatif. Paris : Hachette.

Luyken, G.-M. (1991). Vincre les barrières linguistqiues à la télévision. Doublage et sous-titrage pour un public européen. Manchester : Institut Européen de la Communication.

Pisarska, A., Tomaszkiewicz, T. (1996). Wspótczesne tendencje przekładoznawcze. Poznań : Wydawnictwo Naukowe UAM.

Skibińska, E. (1999). Przekład a kultura. Elementy kulturowe we francuskich thumaczeniach "Pana Tadeusza ». Wrocław : Wydawnictwo Uniwersytetu Wrocławskiego.

Tomaszkiewicz, T. (przekład i adaptacja) (2004). Terminologia tłumaczenia. Poznań : Wydawnictwo Naukowe UAM.

Wojtasiewicz, O. (1992). Wstęp do teorii tlumaczenia. Warszawa : Tepis. 